\title{
Raloxifene improves TNF- $\alpha$-induced osteogenic differentiation inhibition of bone marrow mesenchymal stem cells and alleviates osteoporosis
}

\author{
FENGHE YANG ${ }^{1,2}$, YUSONG JIA ${ }^{3}$, QI SUN ${ }^{3}$, CHENYING ZHENG $^{3}$, CHUYIN LIU $^{3}$, \\ WEI WANG ${ }^{3}, \mathrm{LI} \mathrm{DU}^{3}$, SHENGQIAN KANG $^{3}$, XUFENG NIU ${ }^{1,2}$ and $\mathrm{JINYU} \mathrm{LI}^{3}$

\begin{abstract}
${ }^{1}$ Key Laboratory for Biomechanics and Mechanobiology of Ministry of Education, School of Biological Science Beihang University, Beijing 100083; ${ }^{3}$ Department of Orthopaedics, Dongzhimen Hospital, Beijing University of Chinese Medicine, Beijing 100700, P.R. China
\end{abstract} \\ and Medical Engineering, Beihang University; ${ }^{2}$ Beijing Advanced Innovation Centre for Biomedical Engineering,
}

Received October 11, 2019; Accepted December 9, 2019

DOI: $10.3892 /$ etm.2020.8689

\begin{abstract}
Effect of raloxifene (RLF) on the improvement of inhibited osteogenic differentiation of bone marrow mesenchymal stem cells (BMSCs) resulted from tumor necrosis factor- $\alpha(\mathrm{TNF}-\alpha)$ induction, thus alleviating the progression of osteoporosis (OP), was investigated. An in vivo OP rat model was constructed by performing the procedures of ovariectomy (OVX). Rats were randomly divided into sham group, OVX group and RLF+OVX group. BMSCs were extracted from healthy rats, and randomly divided into control group, TNF- $\alpha$ group, RLF group and TNF- $\alpha+$ RLF group. Viability and cellular calcification ability in each group were detected. The relative levels of osteocalcin (OCN), Runx 2 and NF- $\kappa \mathrm{B}$ in cells with different treatments were determined. The body weight of rats in the OVX group and RLF+OVX group gradually increased compared with that in the sham group on the 8th week. No significant difference in body weight was observed between the rats of the OVX group and RLF+OVX group. Bone metabolism index (BMD) in the rats of the RLF+OVX group was higher than that of the OVX group, and lower compared with that of the sham group. Compared with the sham group, the elastic/max radial degree and elastic/max load of femora were reduced in the OVX group and RLF+OVX group,
\end{abstract}

Correspondence to: Dr Jinyu Li, Department of Orthopaedics, Dongzhimen Hospital, Beijing University of Chinese Medicine, 5 Haiyuncang Alley, Dongcheng, Beijing 100700, P.R. China E-mail: leery_5566@163.com

Dr Xufeng Niu, Key Laboratory for Biomechanics and Mechanobiology of Ministry of Education, School of Biological Science and Medical Engineering, Beihang University, 37 Xueyuan Road, Haidian, Beijing 100083, P.R. China

E-mail: nxf@buaa.edu.cn

Key words: osteoporosis, raloxifene, TNF- $\alpha$ especially in the OVX group. The relative levels of OCN and Runx2, as well as the ALP activity and calcification ability, were decreased in the OVX group compared with the sham group, and the effect was partially reversed by the RLF treatment. After osteogenic differentiation of BMSCs, the viability and calcification ability were markedly reduced in TNF- $\alpha$ group, which was reversed by RLF treatment. Moreover, TNF- $\alpha$ induction downregulated the relative levels of OCN and Runx2, and RLF treatment could enhance their levels. The upregulated NF- $\mathrm{NB}$ protein level, induced by $\mathrm{TNF}-\alpha$, was reduced after RLF treatment. TNF- $\alpha$ induction inhibits osteogenic differentiation of BMSCs, which could be remarkably alleviated by RLF. It is suggested that RLF contributes to the alleviation of OP progression.

\section{Introduction}

Osteoporosis (OP) is a systemic bone disease manifested as low bone mass, destruction of bone microstructure, increased bone fragility and fracture risk (1). The prevalence of OP is on the rise due to the aging of population. It has been reported that in 2010 in China, the prevalence of OP in females $>70$ years of age was $40.0-59.3 \%$, and in males was $14.2-18.9 \%$ (2). OP-induced pain and fracture severely affect life quality and pose economic burden on the elderly (3). It is of significance to focus on the prevention and treatment of OP.

The prevention and treatment strategies of OP are comprehensive, including lifestyle adjustment, calcium and vitamin D supplementation, and application of anti-OP drugs. Estrogen replacement therapy (ERT) can prevent bone loss caused by estrogen deficiency; however, ERT increases the risks of uterine and breast cancer (4). Selective estrogen receptor modulators (SERMs) have been developed as tissue-specific estrogen agonists that are applied for the treatment of postmenopausal OP (5). Raloxifene (RLF) is a second-generation SERM for treating postmenopausal OP which does not have the adverse effects of ERT (6). As an estrogen agonist on bone and several other tissues, RLF suppresses bone loss and reduces fracture risks. In addition, RLF reduces the susceptibility to uterine 
cancer as an estrogen antagonist (7). Currently, RLF has been utilized in the clinical treatment of OP (8); however, the specific pharmacological role of RLF remains to be further explored.

Tumor necrosis factor- $\alpha$ (TNF- $\alpha)$ is a cytokine produced by activated macrophages/monocytes that exerts a crucial role in osteogenic differentiation of stem cells (9). A great number of studies have demonstrated the involvement of TNF- $\alpha$ in mediating multiple pathways related to osteogenic differentiation, such as Wnt, Smads and NF- $\mathrm{B}$ p pathways (10-12). Nevertheless, controversies exist regarding the factors that determine the promotive or inhibitory role of TNF- $\alpha$ in osteogenic differentiation (13). The aim of the present study was to investigate the effect of RLF on TNF- $\alpha$-induced inhibition of osteogenic differentiation and the potential mechanism, and the conclusions of the study may provide new insights for the clinical treatment of OP.

\section{Materials and methods}

Experimental animals. Fifty-four female Sprague-Dawley (SD) rats, 12-weeks old and weighing 238.4 $14 \mathrm{~g}$, were provided by the Shanghai SIPPR-Bk Lab Animal Co., Ltd. Rats were randomly divided into the sham group, ovariectomy (OVX) group and OVX+RLF group, with 18 rats in each group. The rats in the OVX+RLF group were administered with $0.2 \mu \mathrm{M}$ RLF. The body weight of each rat was recorded every week. Rats were housed in a temperature controlled room $\left(21 \pm 2^{\circ} \mathrm{C}\right)$ on a $12: 12$-h light/dark cycle (lights on at 06:00), and all rats had free access to water and food. The study was approved by the Animal Ethics Committee of Beihang University Animal Center (Beijing, China).

Preparation of $O V X$ procedure. Rats were anesthetized by peritoneal administration of $40 \mathrm{mg} / \mathrm{kg}$ pentobarbital sodium. After shaving and skin disinfection, a 2-cm longitudinal incision at $1 \mathrm{~cm}$ near the spine and $2 \mathrm{~cm}$ above posterior iliac crest was made. The abdominal cavity was exposed to resect ovaries. The wound was sutured in layers. Postoperative peritoneal administration of $1 \mathrm{mg} / \mathrm{kg}$ gentamicin was applied. The rats in the sham group were subjected to abdominal cavity exposure and removal of some fat tissues.

MicroCT. After rats were sacrificed, the femoral metaphysis and attached surrounding soft tissues were removed, fixed in $4 \%$ paraformaldehyde solution, and scanned with SCANCO medical microCT (SCANCO Medical AG). Bone histomorphology indicators were determined and analyzed using Image Processing Language (version X; Adobe Systems, Inc.).

Biomechanical examinations. Three-point bending experiment was conducted to record elastic/max radial degree and elastic/max load of rat femora. Briefly, the right femora were placed on the Instron Material Mechanics Testing Device (Instron). At the middle position of femora, a persistent test velocity of $1 \mathrm{~mm} / \mathrm{min}$ was loaded until femoral fracture. Data were recorded and analyzed to obtain the max load.

Isolation and culture of bone marrow mesenchymal stem cells (BMSCs). Rats were sacrificed by cervical dislocation after being anesthetized with pentobarbital sodium via peritoneal administration at a dose of $40 \mathrm{mg} / \mathrm{kg}$. The rats were immersed in $75 \%$ ethanol for $5 \mathrm{~min}$ and isolated for femora and humeri. Rat bones were washed and the epiphysis of long bones was removed. Marrow cavity was repeatedly washed by Dulbecco's modified Eagle's medium (DMEM), and the fluids were inoculated in a $25-\mathrm{ml}$ culture bottle. After incubation for $48 \mathrm{~h}$, the un-adherent cells were washed. Cell passage was performed until $80-90 \%$ confluence. The fourth generation BMSCs were harvested and divided into control group, TNF- $\alpha$ group, RLF group and TNF- $\alpha+\mathrm{RLF}$ group.

Osteogenic differentiation. Fourth generation BMSCs were cultured in a $25-\mathrm{ml}$ culture bottle at $1 \times 10^{7}$ cells/1. BMSCs were subjected to osteogenic differentiation in DMEM (Gibco; Thermo Fisher Scientific, Inc.) containing 10\% fetal bovine serum (Gibco; Thermo Fisher Scientific, Inc.), $10 \mathrm{nmol} / 1$ dexamethasone, $10 \mathrm{mmol} / \mathrm{l} \beta$-glycerophosphate, $50 \mu \mathrm{g} / \mathrm{ml}$ ascorbic acid, $1 \% \mathrm{~L}$-glucose and $1 \%$ penicillin-streptomycin. Osteogenic induction medium was replaced every 2 days.

Reverse transcription-quantitative polymerase chain reaction (RT-qPCR). Total RNA was extracted from BMSCs using TRIzol ${ }^{\circledR}$ reagent (Invitrogen; Thermo Fisher Scientific, Inc.) and the concentration of total RNA was measured using an ultraviolet spectrophotometer (Hitachi, Ltd.). Total RNA was reverse transcribed into cDNA at $50^{\circ} \mathrm{C}$ for $45 \mathrm{~min}$ by using PrimeScript RT reagent kit (Takara Biotechnology Co., Ltd.), according to the manufacturer's protocol. qPCR was subsequently performed using the SYBR-Green Master kit (Roche Diagnostics). The reaction system volume was $25 \mu \mathrm{l}$ in total and the thermocycling conditions were: pre-denaturation at $95^{\circ} \mathrm{C}$ for $5 \mathrm{~min}$, denaturation at $95^{\circ} \mathrm{C}$ for $30 \mathrm{sec}$, annealing at $60^{\circ} \mathrm{C}$ for $45 \mathrm{sec}$, extension at $72^{\circ} \mathrm{C}$ for $3 \mathrm{~min}$, with 35 cycles, and then extension at $72^{\circ} \mathrm{C}$ for $5 \mathrm{~min}$. qPCR products were stored at $4^{\circ} \mathrm{C}$. The relative levels were quantitatively analyzed using the $2^{-\triangle \Delta \mathrm{Cq}}$ method (14). GAPDH was used as internal reference. Primer sequences were as follows: osteocalcin (OCN) forward, 5'-GCCCTGACTGCATTCTGC CTCT-3' and reverse, 5'-TCACCACCTTACTGCCCTCCTG-3'; Runx2 forward, 5'-GGACCGACACAGCCATATAAA-3' and reverse, 5'-GCCTCATTCCCTAACCTGAAA-3'; GAPDH forward, 5'-GCAAGGATACTGAGAGCAAGAG-3' and reverse, 5'-GGATGGAATTGTGAGGGAGATG-3'.

Determination of alkaline phosphatase (ALP) activity. BMSCs were subjected to osteogenic differentiation for 7 days. After cell lysis and centrifugation, the supernatant was harvested for determine the absorbance at $520 \mathrm{~nm}$. The relative ALP activity was calculated based on the protocols of ALP determination kit (Beyotime Institute of Biotechnology).

Alizarin red staining. BMSCs were subjected to osteogenic differentiation for 21 days. Cells were washed with PBS twice, fixed in $4 \%$ paraformaldehyde for $10 \mathrm{~min}$ at $37^{\circ} \mathrm{C}$ and stained with $0.1 \%$ alizarin red $(\mathrm{pH} 4.1)$ for $10 \mathrm{~min}$ at $37^{\circ} \mathrm{C}$. Calcification nodules were observed and captured using a light inverted microscope (magnification, x200; BX-42, Olympus Corporation).

Cell Counting Kit-8 (CCK-8). BMSCs were seeded in a 96-well plate with $3 \times 10^{4}$ cells/well. The absorbance at $450 \mathrm{~nm}$ 
A

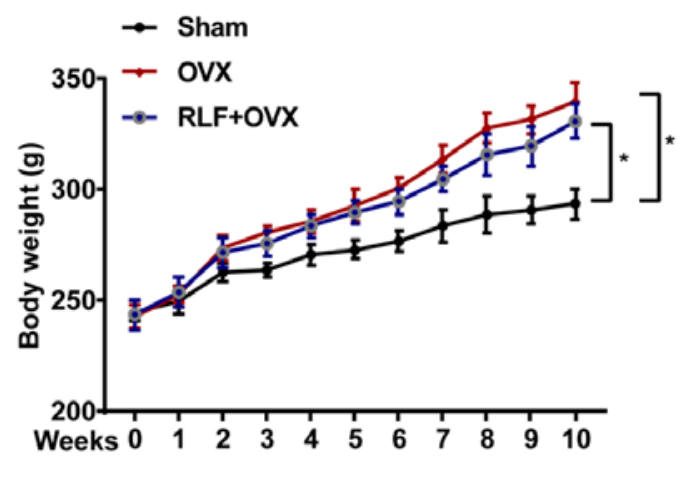

C

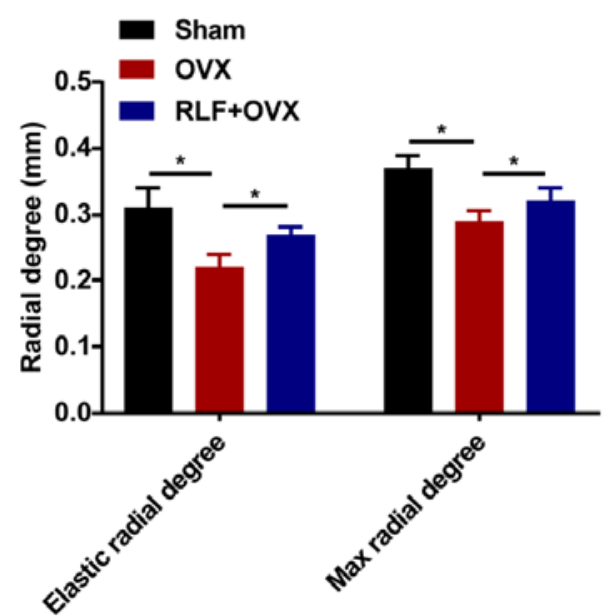

B

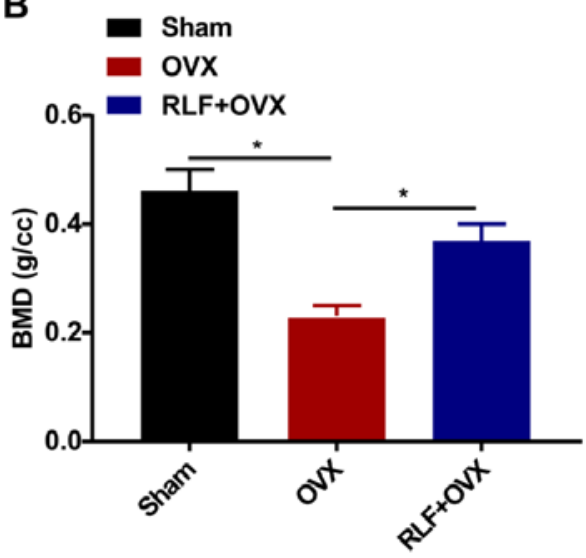

D

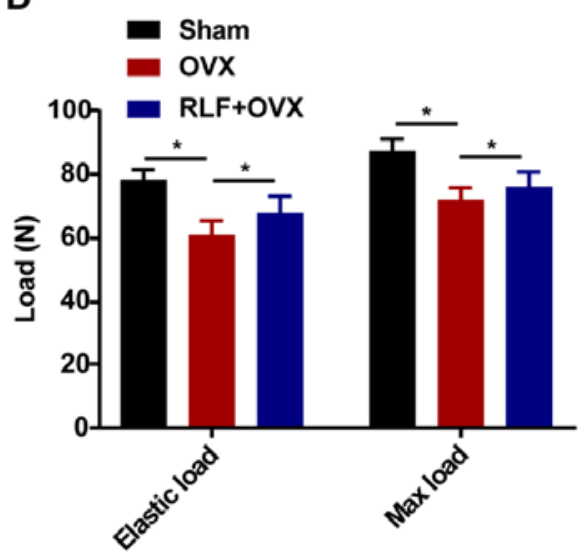

Figure 1. Effect of RLF on body weight, BMD and biomechanical parameters. (A) Body weight of rats of the sham group, OVX group and RLF+OVX group recorded every week. (B) BMD, (C) radial degree and (D) load in rats of the sham group, OVX group and RLF+OVX group. RLF, raloxifene; BMD, bone metabolism index; OVX, ovariectomy. ${ }^{*} \mathrm{P}<0.05$.

was recorded at the appointed time-points using the CCK- 8 kit (Dojindo Molecular Technologies, Inc.) for depicting the viability curve.

Western blot analysis. Total protein was extracted from BMSCs using radioimmunoprecipitation assay and was quantified by bicinchoninic acid (both from Beyotime Institute of Biotechnology) method. A total of $30 \mu \mathrm{g}$ of protein were loaded per lane for electrophoresis. The extracted proteins were separated using a 10\% SDS-PAGE gel. After transferred onto PVDF membranes (EMD Millipore) the proteins were blocked with $5 \%$ skim milk at $20^{\circ} \mathrm{C}$ for $2 \mathrm{~h}$. The membranes were incubated with primary antibodies at $4^{\circ} \mathrm{C}$ overnight and secondary antibodies at $20^{\circ} \mathrm{C}$ for $2 \mathrm{~h}$. Bands were exposed by enhanced chemiluminescence (ECL) detection kit (Amersham; GE Healthcare) and analyzed by ImageJ Software (version 1.38; National Institutes of Health). Rabbit polyclonal NF- $\mathrm{NB}$ antibody (dilution: 1:500; cat. no. ab8805), rabbit polyclonal GAPDH antibody (dilution: 1:500, cat. no. ab37168) and secondary goat anti-rabbit (HRP) IgG antibody (dilution: 1:2,000; cat. no. ab6721) were all purchased from Abcam.

Statistical analysis. SPSS 20.0 statistical software (IBM Corp.) was used for data analysis. Data were expressed as the mean \pm standard deviation. Comparisons between multiple groups were made using one-way ANOVA followed by the Least Significant Difference post hoc test. $\mathrm{P}<0.05$ was considered to indicate a statistically significant difference.

\section{Results}

RLF influences body weight, bone metabolism index (BMD) and biomechanical parameters. Rat body weight was weekly recorded during the whole experiment. All rats in the three groups presented an increased trend in the body weight. Compared with the sham group, the body weight of the rats in the RLF+OVX and OVX groups was gradually elevated in the 8th week. However, no significant difference was observed in the body weight of rats between the RLF+OVX and OVX group (Fig. 1A). In the RLF+OVX group, BMD was higher compared with that in the OVX group and lower than that of the sham group (Fig. 1B). The biomechanical parameters of femoral radial degree and load were also examined. Compared with the sham group, the elastic radial degree/load and max radial degree/load were reduced in the OVX group. However, the RLF treatment markedly increased these biomechanical parameters (Fig. 1C and D).

RLF enhances the osteogenic differentiation ability of BMSCs. The relative levels of OCN and Runx 2 were downregulated in 
A

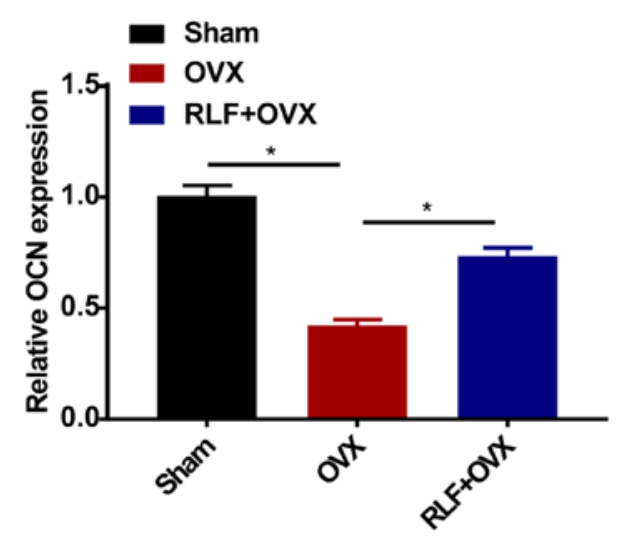

C

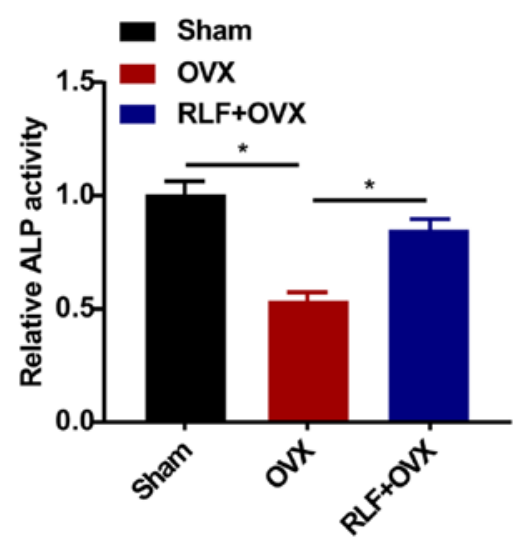

B

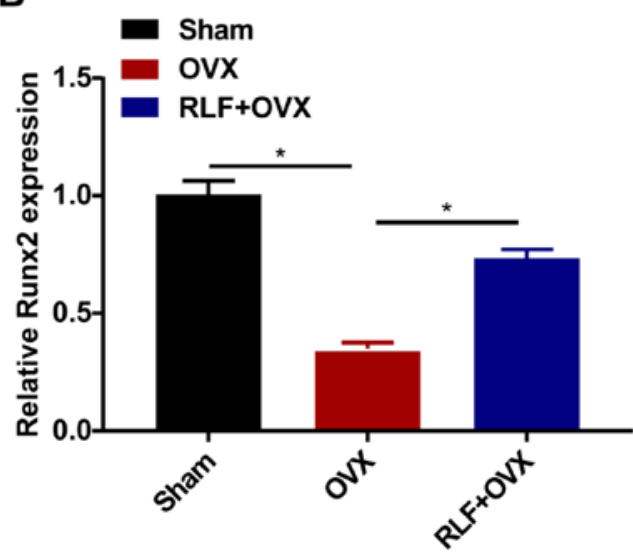

D

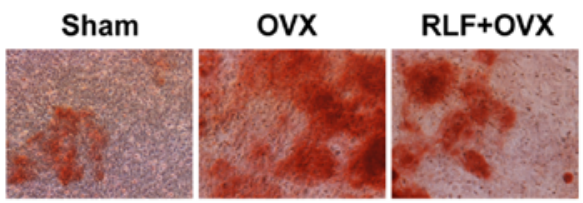

Figure 2. RLF enhanced the osteogenic differentiation ability of BMSCs. Relative levels of (A) OCN and (B) Runx2 in rats of the sham group, OVX group and RLF+OVX group. (C) Relative activity of ALP in rats of the sham group, OVX group and RLF+OVX group. (D) Calcification ability in rats of the sham group, OVX group and RLF+OVX group. RLF, raloxifene; BMSCs, bone marrow mesenchymal stem cells; OCN, osteocalcin; OVX, ovariectomy; ALP, alkaline phosphatase. ${ }^{*} \mathrm{P}<0.05$.

A

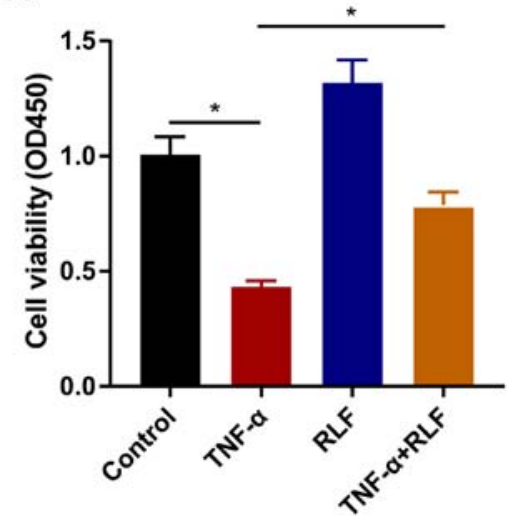

B

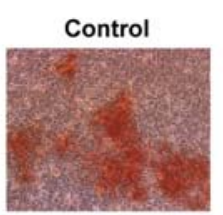

RLF

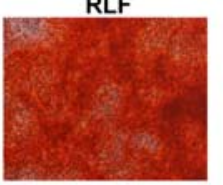

TNF- $\alpha$

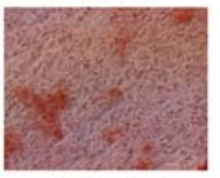

TNF- $a+R L F$

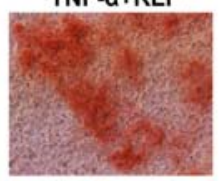

Figure 3. RLF improved the viability and calcification ability of BMSCs. (A) Cell viability and (B) calcification ability in the control group, TNF- $\alpha$ group, RLF group and TNF- $\alpha+$ RLF group. RLF, raloxifene; BMSCs, bone marrow mesenchymal stem cells; TNF- $\alpha$, tumor necrosis factor- $\alpha$. ${ }^{*} \mathrm{P}<0.05$.

the OVX and RLF+OVX group, and especially in the OVX group (Fig. 2A and B). Similarly, the ALP activity was lower in rats undergoing OVX compared with that in rats of the sham group. RLF treatment enhanced ALP activity in OVX rats; however, ALP activity was still lower than that of the sham group (Fig. 2C). Alizarin red staining revealed a pronounced increase in the calcification ability of the RLF+OVX group, indicating the promotive effect of RLF on osteogenic differentiation (Fig. 2D).

$R L F$ improves viability and calcification ability of BMSCs. To further uncover the in vitro effects of RLF on osteogenic differentiation of BMSCs, the primary BMSCs were divided into four groups based on different treatments. Cell viability markedly 

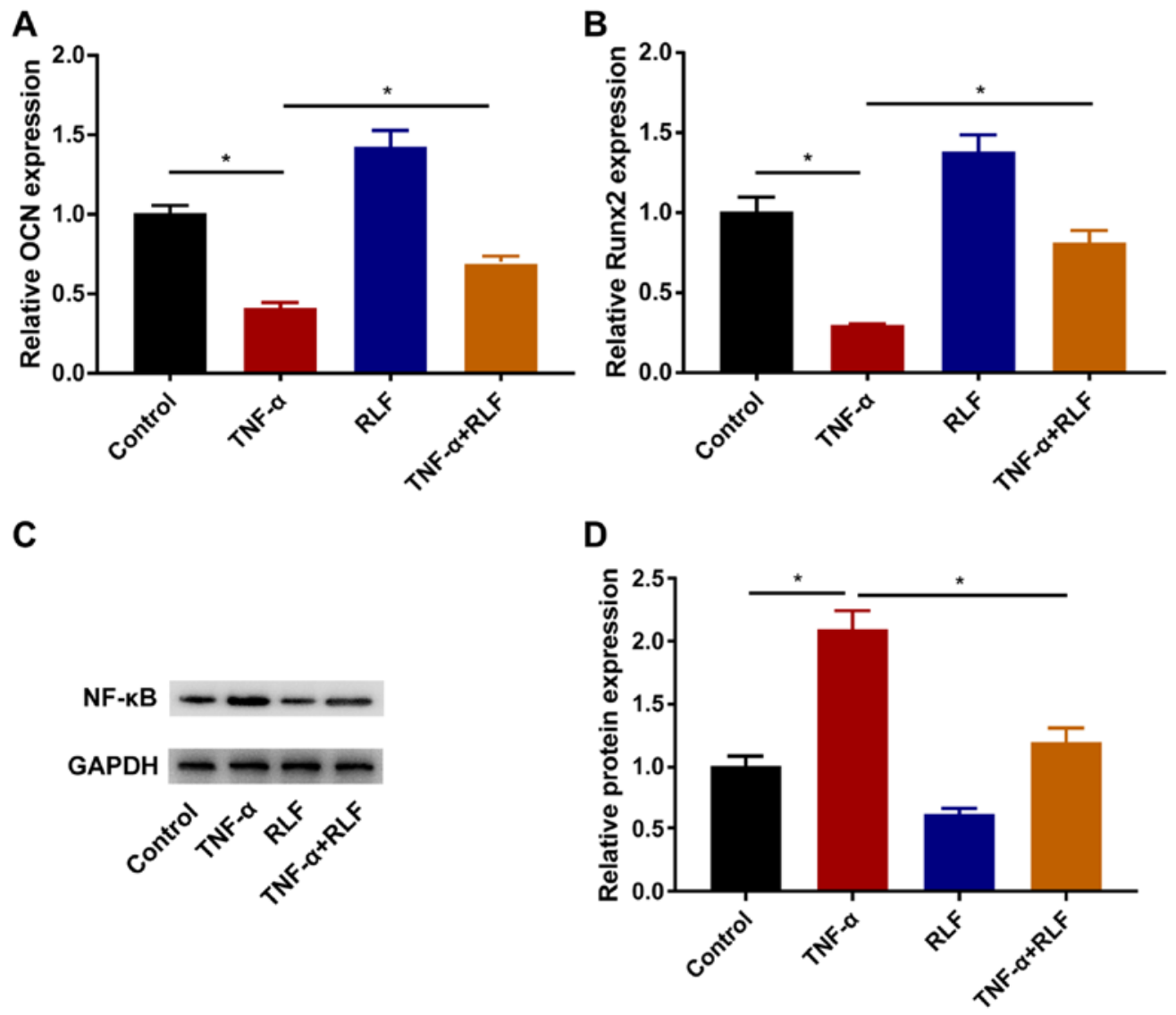

Figure 4. RLF regulated the osteogenesis-related genes and the NF- $\mathrm{kB}$ pathway. Relative levels of (A) OCN and (B) Runx2 in the control group, TNF- $\alpha$ group, RLF group and TNF- $\alpha+$ RLF group. (C) Western blot analysis and (D) protein levels of NF- $\kappa B$ in the control group, TNF- $\alpha$ group, RLF group and TNF- $\alpha+R L F$ group. RLF, raloxifene; OCN, osteocalcin; TNF- $\alpha$, tumor necrosis factor- $\alpha$. "P<0.05.

decreased after TNF- $\alpha$ induction in BMSCs. Conversely, RLF treatment could promote their viability. Notably, RLF treatment accelerated the viability in TNF- $\alpha$-induced BMSCs (Fig. 3A). Furthermore, the RLF treatment stimulated the calcification ability in BMSCs; however, the calcification ability was markedly inhibited by TNF- $\alpha$ induction (Fig. 3B).

$R L F$ regulates osteogenesis-related genes and the $N F-\kappa B$ pathway. TNF- $\alpha$ induction downregulated OCN and Runx2 in BMSCs, which effect was partially reversed after the RLF treatment (Fig. 4A and B). The protein level of NF- $\mathrm{B}$ was determined in each group. After TNF- $\alpha$ induction, the protein level of $\mathrm{NF}-\kappa \mathrm{B}$ was remarkably upregulated, which was suppressed by the RLF treatment (Fig. 4C and D).

\section{Discussion}

Bones maintain structural stability through sustained bone remodeling and resorption. Bone tissues include osteoblasts, osteoclasts, and osteocytes. The osteogenic capacity of osteoblasts and the bone resorption function of osteoclasts maintain the balance of bone remodeling. Once the balance breaks, it leads to bone diseases, such as OP (14). OP is common in the elderly and enhances the fracture risks. It is estimated that $72 \%$ of females and $62 \%$ of males $>50$ years of age will suffer from OP or osteopenia in 2022 (15). OP-induced fractures pose a huge economic burden on the society (16).
ERT markedly alleviates bone mass reduction and reduces the incidence of fracture. However, several large-scale clinical trials have proposed that ERT increases the susceptibility to invasive breast cancer, coronary disease, stroke and pulmonary embolism (17). Selective estrogen receptor modulators (SERMs) have attracted attention because of their estrogen agonistic or antagonistic effects. RLF, the new generation of SERMs, has been applied as an ideal drug for postmenopausal OP (18).

Cytokines can stimulate the development of osteoclasts in the presence of stromal cells or osteoblasts, including IL-1 $\beta$, IL-1, IL- 6 and TNF- $\alpha$. It has been suggested that these cytokines may indirectly induce osteoclast formation through autocrine or paracrine modes (19). TNF is mainly synthesized by mononuclear macrophages, and can also be secreted by T cells, natural killer cells, chondrocytes, and osteoblasts (20). Roggia et al (21) have pointed out the rapid bone loss in OVX mice, whereas TNF- $\alpha$ deficiency mice do not present such a change, indicating the crucial role of TNF- $\alpha$ in the development of OP induced by ovarian function decline. It has been reported that estrogens suppress the expression of TNF- $\alpha$ in monocytes (22). Consistently, this study demonstrated the inhibitory effect of TNF- $\alpha$ on osteogenic differentiation, which was reversed by RLF treatment through the NF- $\kappa$ B pathway.

In conclusion, TNF- $\alpha$ induction inhibits osteogenic differentiation of BMSCs, which could be remarkably alleviated by RLF. It is suggested that RLF contributes to the alleviation of OP progression. 


\section{Acknowledgements}

Not applicable.

\section{Funding}

The study was supported by the National Natural Science Foundation of China (81503601), the Young Talents Training Program of Dongzhimen Hospital of the Beijing University of Chinese Medicine (DZMYS-201802), the Basic Scientific Research Service Fee project of the Beijing University of Chinese Medicine (2015-JYB-JSMS080, 2016-JYB-JSMS-045) and the 111 Project (no. B13003).

\section{Availability of data and materials}

All data generated or analyzed during this study are included in this published article.

\section{Authors' contributions}

FY, JL and XN designed the study and performed the experiments. FY, YJ and QS established the animal model. CZ and $\mathrm{CL}$ acquired the data and were also involved in the conception of the study. WW, LD and SK analyzed the data. FY, JL and $\mathrm{XN}$ prepared the manuscript. All authors read and approved the final manuscript.

\section{Ethics approval and consent to participate}

The study was approved by the Animal Ethics Committee of Beihang University Animal Center (Beijing, China).

\section{Patient consent for publication}

Not applicable.

\section{Competing interests}

The authors declare that they have no competing interests.

\section{References}

1. Hu BT and Chen WZ: MOTS-c improves osteoporosis by promoting osteogenic differentiation of bone marrow mesenchymal stem cells via TGF- $\beta /$ Smad pathway. Eur Rev Med Pharmacol Sci 22: 7156-7163, 2018.

2. Zhu H, Fang J, Luo X, Yu W, Zhao Y, Li X, Du J and Lu Y: A survey of bone mineral density of healthy Han adults in China. Osteoporos Int 21: 765-772, 2010.

3. Hernlund $\mathrm{E}$, Svedbom A, Ivergård $\mathrm{M}$, Compston $\mathrm{J}$, Cooper $\mathrm{C}$, Stenmark J, McCloskey EV, Jönsson B and Kanis JA: Osteoporosis in the European Union: Medical management, epidemiology and economic burden. A report prepared in collaboration with the International Osteoporosis Foundation (IOF) and the European Federation of Pharmaceutical Industry Associations (EFPIA). Arch Osteoporos 8: 136, 2013.

4. Chavassieux P, Portero-Muzy N, Roux JP, Garnero P and Chapurlat R: Are biochemical markers of bone turnover representative of bone histomorphometry in 370 postmenopausal women? J Clin Endocrinol Metab 100: 4662-4668, 2015.

5. Rodan GA and Martin TJ: Therapeutic approaches to bone diseases. Science 289: 1508-1514, 2000.
6. Brennan TC, Rizzoli R and Ammann P: Selective modification of bone quality by PTH, pamidronate, or raloxifene. J Bone Miner Res 24: 800-808, 2009.

7. Lamas AZ, Caliman IF, Dalpiaz PL, de Melo AF Jr, Abreu GR, Lemos EM, Gouvea SA and Bissoli NS: Comparative effects of estrogen, raloxifene and tamoxifen on endothelial dysfunction, inflammatory markers and oxidative stress in ovariectomized rats. Life Sci 124: 101-109, 2015.

8. Ettinger B, Black DM, Mitlak BH, Knickerbocker RK, Nickelsen T, Genant HK, Christiansen C, Delmas PD, Zanchetta JR, Stakkestad J, et al; Multiple Outcomes of Raloxifene Evaluation (MORE) Investigators: Reduction of vertebral fracture risk in postmenopausal women with osteoporosis treated with raloxifene: Results from a 3-year randomized clinical trial. JAMA 282: 637-645, 1999.

9. Ding J, Ghali O, Lencel P, Broux O, Chauveau C, Devedjian JC, Hardouin P and Magne D: TNF-alpha and IL-1beta inhibit RUNX2 and collagen expression but increase alkaline phosphatase activity and mineralization in human mesenchymal stem cells. Life Sci 84: 499-504, 2009.

10. Qiu W, Andersen TE, Bollerslev J, Mandrup S, Abdallah BM and Kassem M: Patients with high bone mass phenotype exhibit enhanced osteoblast differentiation and inhibition of adipogenesis of human mesenchymal stem cells. J Bone Miner Res 22: 1720-1731, 2007.

11. Mukai T, Otsuka F, Otani H, Yamashita M, Takasugi K, Inagaki K, Yamamura M and Makino H: TNF-alpha inhibits BMP-induced osteoblast differentiation through activating SAPK/JNK signaling. Biochem Biophys Res Commun 356: 1004-1010, 2007.

12. Chang J, Liu F, Lee M, Wu B, Ting K, Zara JN, Soo C, Al Hezaimi K, Zou W, Chen X, et al: NF- $\kappa \mathrm{B}$ inhibits osteogenic differentiation of mesenchymal stem cells by promoting $\beta$-catenin degradation. Proc Natl Acad Sci USA 110: 9469-9474, 2013.

13. Gilbert L, He X, Farmer P, Rubin J, Drissi H, van Wijnen AJ, Lian JB, Stein GS and Nanes MS: Expression of the osteoblast differentiation factor RUNX2 (Cbfa1/AML3/Pebp2alpha A) is inhibited by tumor necrosis factor-alpha. J Biol Chem 277 : 2695-2701, 2002.

14. Livak KJ and Schmittgen TD: Analysis of relative gene expression data using real-time quantitative PCR and the 2(-Delta Delta C(T)) Method. Methods 25: 402-408, 2001.

15. Kemmler W, Häberle L and von Stengel S: Effects of exercise on fracture reduction in older adults: A systematic review and meta-analysis. Osteoporos Int 24: 1937-1950, 2013.

16. Harvey N, Dennison E and Cooper C: Osteoporosis: Impact on health and economics. Nat Rev Rheumatol 6: 99-105, 2010.

17. Rossouw JE, Anderson GL, Prentice RL, LaCroix AZ, Kooperberg C, Stefanick ML, Jackson RD, Beresford SA, Howard BV, Johnson KC, et al; Writing Group for the Women's Health Initiative Investigators: Risks and benefits of estrogen plus progestin in healthy postmenopausal women: Principal results From the Women's Health Initiative randomized controlled trial. JAMA 288: 321-333, 2002.

18. Passeri G, Girasole G, Jilka RL and Manolagas SC: Increased interleukin- 6 production by murine bone marrow and bone cells after estrogen withdrawal. Endocrinology 133: 822-828, 1993.

19. Vitale RF and Ribeiro FA: The role of tumor necrosis factor-alpha (TNF-alpha) in bone resorption present in middle ear cholesteatoma. Rev Bras Otorrinolaringol (Engl Ed) 73: 117-121, 2007.

20. Rayl JM, Wellehan JFX Jr, Bunick D and Allender MC: Development of reverse-transcriptase quantitative PCR assays for detection of the cytokines IL-1 $\beta$, TNF- $\alpha$, and IL-10 in chelonians. Cytokine 119: 16-23, 2019.

21. Roggia C, Gao Y, Cenci S, Weitzmann MN, Toraldo G, Isaia G and Pacifici R: Up-regulation of TNF-producing T cells in the bone marrow: A key mechanism by which estrogen deficiency induces bone loss in vivo. Proc Natl Acad Sci USA 98: 13960-13965, 2001

22. An J, Tzagarakis-Foster C, Scharschmidt TC, Lomri N and Leitman DC: Estrogen receptor beta-selective transcriptional activity and recruitment of coregulators by phytoestrogens. J Biol Chem 276: 17808-17814, 2001. International (CC BY-NC-ND 4.0) License. 